\title{
G

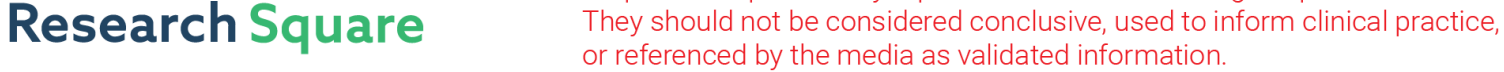 \\ Intramolecular Orthogonal Reporters for Dissecting Oxidative Stress and Response
}

\section{Yecan Pan}

Institute of Quality Standard and Testing Technology for Agro-Products, Chinese Academy of Agricultural Sciences

\section{Rui Weng}

Institute of Quality Standard and Testing Technology for Agro-Products, Chinese Academy of Agricultural Sciences

\section{Linghao Zhang}

Beijing University of Chemical Technology

\section{Jing Qiu}

Institute of Quality Standard and Testing Technology for Agro-Products, Chinese Academy of Agricultural Sciences

\section{Xinlu Wang}

Institute of Quality Standard and Testing Technology for Agro-Products, Chinese Academy of Agricultural Sciences

\section{Guangqin Liao}

Institute of Quality Standard and Testing Technology for Agro-Products, Chinese Academy of Agricultural Sciences

\section{Zhaohui Qin}

Beijing University of Chemical Technology

\section{Rui Liu}

Tsinghua University

\section{Xiaochuan Dai}

Tsinghua University

\section{Yongzhong Qian}

Institute of Quality Standard and Testing Technology for Agro-Products, Chinese Academy of Agricultural Sciences

Xin Su ( $\sim$ pkusuxin@gmail.com )

Beijing University of Chemical Technology https://orcid.org/0000-0002-6629-9856

\section{Article}

Keywords: MD simulation, DNA nanotechnology, Fluorescence imaging, Reactive oxygen species (ROS), apurinic/apyrimidinic endonuclease, in vivo imaging, Allicin 
Posted Date: October 21st, 2021

DOI: https://doi.org/10.21203/rs.3.rs-917337/v1

License: (c) (i) This work is licensed under a Creative Commons Attribution 4.0 International License. Read Full License 


\section{Abstract}

Molecular response under oxidative stress is a well-known reflection of the development of various diseases. Fluorescent probes for reactive oxygen species (ROS) and for their related molecules integrated into single nanostructures could simultaneously map the localization and abundance of multiple targets with spatiotemporal accuracy in live cells. However, it is extremely challenging because proximity-induced fluorophore interaction leads to low sensitivity and poor specificity. Herein, we engineered intramolecular Orthogonal Reporters (iOR) based on DNA nanostructure scaffolds. Fluorescence reporters in iOR were precisely located with appropriate distance achieved by MD simulation. iOR functionalized with nuclear localization signal peptide is capable of imaging ROS and its related gene repair enzyme (APE1) in nucleus and cytoplasm with spatiotemporal accuracy. iOR discloses the strong positive relation of ROS and APE1 as well as their synergistic regulation. In vivo fluorescence imaging with iOR enables direct observation of ROS and APE1 of the tumor sites of mice. iOR represents a new concept of molecule probes for simultaneous detection of multiple related targets in living cells. We anticipate this simulationguided probe to find broad applications in cellular biology and nanotechnology.

\section{Introduction}

Oxidative stress resulting from effects of reactive oxygen species (ROS) such as superoxide $\left(\mathrm{O}_{2}{ }^{-{ }^{-}}\right)$, hydrogen peroxide $\left(\mathrm{H}_{2} \mathrm{O}_{2}\right)$, and the hydroxyl radical $(\mathrm{OH} \cdot)$ plays an important role in the pathogenesis of a variety of diseases and important biological processes ${ }^{1,2}$. Toxic effects of oxidative stress lead to cellular damage by oxidizing nucleic acids, proteins, and membrane lipids. In mammalian cells, oxidative stress and the corresponding molecular responses are thought to be linked to a variety of diseases such as cancer ${ }^{3}$, Parkinson's disease ${ }^{4}$, Alzheimer's disease ${ }^{5}$, heart failure ${ }^{6}$, and even cardiac complications in COVID-197 . Particularly, oxidative stress-induced cancer development is mainly attributed to the direct DNA damage caused by ROS, which is mutagenic, and may also suppress apoptosis and promote proliferation, invasiveness and metastasis ${ }^{8}$. DNA damage results in the accumulation of excessive apurinic/apyrimidic sites (AP sites) thereby activating base excision pathway for DNA repair ${ }^{9}$. On the other hand, ROS-induced DNA damage can be beneficial, as they are utilized for cancer therapeutics, such as photodynamic therapy, which employ the oxidative stress and response to elicit cell death ${ }^{10,11}$. Therefore, it is of great significance to quantitatively map cellular ROS and the DNA repair enzymes for ROS administration and utilization. This motivates the development of advanced tools to observe ROS and its connected molecules with spatiotemporal accuracy in live cells.

Fluorescence imaging emerges as a powerful tool for directly observing the localizations and activities of labeled biomolecules despite the crowded intracellular environment. It is desired to integrate molecular probes into single nanostructures to simultaneously map multiple targets in live cells ${ }^{13,14}$. Such design enables to study the colocalization and abundance of multiple targets with spatiotemporal accuracy. However, uncontrolled integration of multiple fluorescent reporters into a single assembly leads to unexpected interaction between fluorophores resulting in low sensitivity and poor target specificity. 
Employing the base-pairing language to construct nanostructures in a programmable manner, structural DNA nanotechnology has been widely applied in biosensors, molecular logic devices, and drug delivery vehicles $^{15-17}$. The well-defined geometry of DNA nanostructure makes them promising scaffolds to organize probe molecules in an addressable manner and integrate distinct functions in precise stoichiometries improving the specificity, sensitivity, and throughput of biosensors ${ }^{15}$. The high biocompatibility of DNA nanostructures enables them to enter cells autonomously ${ }^{18,19}$. DNA nanodevices are versatile chemical reporters that can quantitatively map multiple targets in living systems in real time ${ }^{18,19}$. However, it remains challenging to eliminate crosstalk between probes even by using DNA nanodevices because the flexibility of DNA sequence and nanostructures may induce unexpected fluorophore interaction.

Here, we reported intramolecular Orthogonal Reporters (iOR) based on DNA nanostructure scaffolds in which fluorescence reporters were precisely located with appropriate distance by the assistance of MD simulation. Spectrally separated fluorophores in iOR respond to their corresponding targets with superior specificity. iOR functionalized with Nuclear targeting signal (NLS) peptide (NiOR) is capable of surfing in both the cytoplasm and nucleus. NiOR quantitatively maps ROS and its induced overexpression of gene repair enzyme apurinic/apyrimidic (AP) endonuclease 1 (APE1) in living cells with spatiotemporal accuracy. Fluorescence imaging discloses that the expression level of APE1 is proportional to ROS level, and that their nuclear and mitochondrial accumulation are regulated by oxidative agents and natural antioxidants. In vivo fluorescence imaging of tumor-bearing mice by NiOR also demonstrate the strong correlation between ROS and APE1 and the regulatory effect of those external stimuli. We anticipated that this simulation-guided iOR represented a new class of molecular probes for cellular imaging applications providing opportunities for deep understanding of the molecule interplay in live cells.

\section{Results}

\section{MD simulation-guided design of iOR.}

We chose DNA tetrahedron as the principal scaffold for iOR owing to its excellent biocompatibility and structural simplicity ${ }^{21}$. The modularity of DNA tetrahedron enables for integrating the fluorescence reporters of ROS and APE1 (Scheme 1). Dihydroethidium (DHE) was chosen as the ROS reporter since it could emit fluorescence at $590 \mathrm{~nm}$ when oxidated by superoxide $\mathrm{O}_{2}{ }^{-{ }^{-}}$. The fluorescence has been used increasingly for fluorescent analysis of ROS output in cells and tissues as it shows a linear relation with $\mathrm{O}_{2}{ }^{\cdot-}$ concentration (Figure S1A and B). ${ }^{22}$. APE1 specifically cleaves AP site and therefore plays a significant role in BER pathway, particularly in the ROS-regulated BER ${ }^{23}$. Abnormal expression and subcellular distribution of APE1 have been linked to tumor development ${ }^{24,25}$. As our previous study, the simple fluorescence dequenching of nucleic acid probes can be used for monitoring and quantifying the enzyme activity ${ }^{26,27}$. As shown in Scheme 1, the cleavage of AP site by APE1 results in the separation of Cy5 dye and $\mathrm{BHQ} 3$ quencher, leading to fluorescence restoration. APE1 reporter exhibits a linearity to APE1 concentration (Figure S2A and B), and gel electrophoresis also confirmed its correct function 
(Figure S2C). Although DHE and Cy5 are separated in emission profiles, the overlap of the emission profile of DHE and the excitation profile of Cy 5 would lead to Förster resonance energy transfer (FRET) between the two fluorophores which results in poor sensitivity and specificity. Accordingly, it is desired to precisely control the distance between the fluorophores to avoid FRET.

IOR was designed in silico by Tiamat software, and MD simulation were performed to optimize the structures (Figure 2A). oxDNA, a coarse-gained model to incorporate thermodynamics as parameterized by the nearest-neighbor model into a description with continuous degrees of freedom that captures structural and mechanical properties of DNA ${ }^{29}$. oxDNA has previously been used to successfully assist the optimization of a variety of DNA nanostructures ${ }^{29}$. With oxDNA, the molecular behavior and structural flexibility of iOR candidates were simulated, and the distances between the two fluorophores in different candidates were evaluated (Figure 2B), the simulation parameters were noted in Table S2. The average distances increase as a function of polyT spacer length. Due to the flexibility of the DNA strand, the deviation of distance distribution also increases with the spacer length. The Förster distance of DHE and Cy5 was estimated as $5.4 \mathrm{~nm}$ which means the FRET efficiency can be lower than $10 \%$ if the distance between dyes exceeds $10 \mathrm{~nm}^{33}$. Therefore, based on the simulated dye distance distribution, the probabilities for $10 \%$ FRET of the iOR candidates can be obtained (Figure 2B). S1-0T and S2-0T exhibits relatively low FRET probability among the candidates. By comprehensively considering the average distance of dyes and the FRET probability, S1-0T was chosen for potential iOR.

\section{iOR assemble and characterization}

DHE dye was labeled on the carboxyl-modified ssDNA via NHS/EDC chemistry (Figure S3A), the DHEmodified SsDNA shows slower electrophoresis speed indicating the successful modification (Figure S3B). Next, DHE-modified strand (P7-DHE, see Table S1), APE1 reporter strands (P5 and P6, see Table S1), and DNA tetrahedron scaffold strands (P1, P2, P3, and P4, see Table S1) were assembled to formulate iOR. The stepwise strand assembly was verified by $6 \%$ non-denaturing polyacrylamide gel electrophoresis (PAGE) (Figure 2C), the single band in lane 7 indicates the successful assembly and high purity of iOR. The nanostructure was characterized by AFM image showing the size is $\sim 14 \mathrm{~nm}$ (Figure 2D). Dynamic light scattering (DLS) also indicated the average hydrodynamic size of iOR is $16.2 \mathrm{~nm}$ (Figure S4). Next, the ROS response of the optimized iOR candidate (S1-OT) was tested showing the greatly enhanced DHE fluorescence as well as low FRET efficiency of $17.2 \%$ in the presence of ROS. In contrast, high FRET efficiency of $52.1 \%$ was found by using an undesired iOR candidate S3-0T resulting in weak fluorescence of FRET donor DHE and poor sensitivity for ROS (Figure 2E). These results highlight the accuracy of MD simulation of potential iOR structures.

The sensitivity and orthogonality were evaluated as a function of ROS and APE1. The two reporters show a similar linearity to their corresponding targets allowing for a detection limit of $330 \mathrm{nM}$ and $5 \mathrm{pM}$ for ROS and APE1, respectively. Across various concentrations of each target, iOR specifically produced fluorescence that responded only to respective targets, thereby enabling orthogonal dual detection of two targets (Figure 1F). Non-specific nucleases which may induce nonspecific signal of iOR are commonly 
found in biological fluids as well as in live cells ${ }^{34,35}$. As previously reported, DNA nanostructures may protect the carried nucleic acid probes from non-specific degradation because the rigid scaffold may restrict enzyme activity ${ }^{19}$. As expected, there is no significant fluorescence increase in the presence of other nucleases (Figure S5A and B).

\section{Nucleus targeting iOR and its internalization}

Owing to its superior orthogonality, IOR was operated in living cells for simultaneously imaging ROS and APE1. DNA tetrahedrons can be internalized by in mammalian cells via a lipid-raft-/caveolin-mediated endocytic pathway ${ }^{36}$. Nevertheless, such nanostructures do not have the capability of nucleus entry. To study ROS and APE1 in nucleus, IOR was functionalized with NLS peptide which facilitated the cellular uptake of nanoparticles toward nucleus in a highly ordered manner with the help of molecular motors and reduced the possibility to the lysosomes ${ }^{37,38}$. iOR was conjugated with NLS by click chemistry. As shown in Figure 2A, azide-labeled oligonucleotide reacted with alkyne-labeled NLS at room temperature. The successful conjugation is indicated in the MALDI mass spectrum by the appearance of a signal corresponding to a compound with a mass equal to the sum of the masses of the peptide and the oligonucleotide of 9496.53 (Figure 2B). The conjugate product was integrated with iOR by SsDNA hybridization. As shown in Figure S6, gel electrophoresis indicates the successful modification of NLS on iOR (NiOR), with the bands at different locations. Both iOR and NiOR were singly labeled with Cy5 to study their cellular distribution. Hela cells incubated with NLS functionalized iOR (NiOR) exhibits significantly 5.1 times higher fluorescence intensity in nucleus than that incubated with iOR. It indicates that NLS functionalization can effectively deliver iOR into nucleus (Figure 2C). Both NiOR and iOR are distributed thoroughly in cytoplasm owing to the capability of DNA nanostructures for endo-lysosomal escape (Figure 2C). Furthermore, the biocompatibility of probes was tested with the use of iOR and NiOR for 2-4 h. All groups show over $90 \%$ cellular viability indicating the high biocompatibility of these molecular probes.

NiOR was applied for the simultaneous imaging of ROS and APE1 in Hela cancer cells. The fluorescence of DHE and Cy 5 gradually increases as a function of time suggesting that NiOR can be internalized efficiently within $2 \mathrm{~h}$, and the reactions between the reporters and their corresponding targets are sufficiently sensitive to detect endogenous ROS and APE1 (Figure 2D and E). Since ROS and APE1 reporters are internalized via the same DNA scaffold, they would be expected to co-distribute in nucleus and cytoplasm following internalization. Owing to the function of NLS, nuclear and cytoplasmic ROS and APE1 can be successfully detected by NiOR.

\section{Dissecting the regulation and subcellular distribution of cellular ROS and APE1 by NiOR.}

Abundance, detailed subcellular distribution, and corresponding regulation of ROS and APE1 were further investigated (Figure 3). The expression level of DNA repair enzyme APE1 is expected to relate to oxidative stress because they protect DNA from oxidation damage. Since cancer cells are typically growing vigorously, they have an elevated demand for energy production and, thus, generate a high level of 
ROS $^{37,38}$. We analyzed ROS and APE1 in two cancer cell lines HeLa and A549 as well as one normal cell line HEK-293 (Figure 3A and Figure S8). The abundance of cytoplasmic and nuclear APE1 of cancer lines is significantly higher than that of normal cell line as ROS shows the same trend. More specifically, the expression of APE1/ROS in HeLa cytoplasm was 2.6/1.8 times that of HEK-293, and about 3.1/1.7 times for A549. The expression of APE1/ROS in HeLa nucleus was 2.8 / 2.5 times as much as HEK-293, and 3.6 / 4.1 were due to A549 (Figure S8B). These results indicate that the expression level of APE1 is strongly correlated to ROS abundance. In these cancer cells, due to changes in angiogenesis, there are cycles of hypoxia/re-oxygenation that contribute to a high ROS level. Responses to increased ROS include the elevated level of APE1, which might be responsible to maintain DNA integrity and function ${ }^{40,41}$. Mitochondria is responsible to process an extra-nuclear genome such as mtDNA, which is highly susceptible to attack by ROS. In the cells with high metabolic or proliferative rates, APE1 was expected to display co-localization with mitochondria ${ }^{42,43}$. To test this hypothesis, we used mitochondrial tracker to label mitochondria. APE1 and ROS exhibit Pearson's correlation coefficient (PCC) of 0.68 and 0.65 for mitochondria, respectively, and PCC of APE1 and ROS is 0.69 , suggesting that three targets are slightly correlated in localization in the absence of external stimuli (Figure 3B).

The regulation of ROS and APE1 by external stimuli was studied. Phorbol myristate acetate (PMA) is believed to stimulate oxidative burst and release of ROS in various types of mammalian cell resulting in imbalance and abnormality at molecular level ${ }^{44-46}$. HeLa cells were stimulated to produce $\mathrm{O}_{2}{ }^{--}$by PMA in advance, and the fluorescence of DHE and Cy5 of NiOR were enhanced simultaneously, implying the level of cytoplasmic and nuclear APE1/ROS were 1.7/1.7 and 2.0/4.3 times elevated with the simulation of PMA (Figure 3A and 3C). PCC value of ROS and APE1 increased to 0.91 yielding a strong spatial correlation. Meanwhile, the colocalization of ROS/mitochondria and APE1/mitochondria was also enhanced (Figure 3B). These results suggest that nuclear APE1 is overexpressed and cytoplasmic APE1 is accumulated under elevated oxidative stress to maintain the integrity of genomic DNA and mtDNA. To further confirm this mechanism, we extracted cellular DNA and measured the degree of guanine oxidation by ELISA (Figure S9A), and found that oxidized guanine (8-oxo-dG) significantly increased by $29 \%$ as PMA stimulation (Figure S9B), which supports the proposed mechanism. Allicin [S-(2-propenyl)-2propene-1-sulfinothioate], the most biologically active sulfur-containing compound of garlic, was reported to be capable of modulating cellular ROS and increasing cellular antioxidant enzymes ${ }^{47}$. Allicin an organosulfur antioxidant obtained from garlic was proposed to be capable of effectively protecting cells from the adverse effects of oxidative stress ${ }^{48,49}$. As shown in Figure 3A (row 3) and 3C, the level of ROS and APE1 was reduced when cells were treated with allicin. Moreover, allicin can effectively weaken the fluorescence of ROS and APE1 in PMA stimulated cells, and allicin pre-treated cell is less sensitive to PMA simulation (row 4 and row 5 in Figure $3 A$ and $3 C$ ). Allicin also down regulated the level of 8-oxo-dG in the untreated cells and the PMA-stimulated cells (Figure S9B). All these results indicate that allicin effectively mitigates cellular oxidative stress and protects cells from oxidative simulation, which is consistent with the previous observations. 
The western blots performed show good agreement with the results of NiOR and validate the expression level of APE1 under different conditions. (Figure S10). Commercially available ROS probe (DCFH-DA) was used to test ROS level, also indicating PMA and allicin can up and down regulate ROS, respectively (Figure S11). All these results demonstrate that NiOR correctly reflects the subcellular distribution of ROS and APE1 as well as their regulation by external stimuli.

\section{In vivo fluorescence imaging of tumor-bearing mice}

Encouraged by the successful fluorescence imaging of NiOR in living cells, we further evaluated the ability of NiOR to function in animals. The in vivo imaging was performed through in situ injection of $\mathrm{NiOR}$ into BALB/c mice bearing subcutaneous HeLa cervical cancer xenografts (Figure 4A). After 4h of injection, fluorescence imaging was performed. As shown in Figure 4B, fluorescence signal of DHE and Cy5 accumulated at the tumor site, implying that NiOR can reflect the level of ROS and APE1 accordingly. The DHE and Cy 5 signal of all groups reach maximum at $1 \mathrm{~h}$ and $2 \mathrm{~h}$ after injection, respectively (Figure $4 \mathrm{D})$. After euthanizing the mice at $24 \mathrm{~h}$ post-injection, the major organs and tumors were harvested for $e x$ vivo imaging suggesting that NiOR was massively accumulated at the tumor site (Figure 4C, 4E and Figure S12). Lipopolysaccharides (LPS) was commonly used to elevate the ROS level in experimental mice $^{50}$. As expected, under LPS stimulation, the fluorescence of DHE and Cy 5 enhanced simultaneously attributed to the increased ROS and induced overexpressed APE1 (Figure 4B), ex vivo imaging of harvested tumor also supported this effect. The function of allicin to mitigate oxidative stress was also validated on both the control mice and LPS simulated mice (Figure 4B and $4 \mathrm{C}$ ). The fluorescence of DHE and Cy 5 of allicin-treated mice was lower than the corresponding untreated groups (Figure 4D). Collectively, the molecular probe NiOR can be successfully deployed in experimental mice, and the results are consistent with cell imaging. In addition, immunohistochemistry validated the fact that LPS and allicin are capable of up- and down-regulating APE1 on mice, respectively, which was the important verification evidence for NiOR to detect the expression of APE1 in vivo (Figure S13). TUNEL staining revealed that all groups exhibit a low level of nucleus necrosis and apoptosis of tumor tissue (Figure S14). H\&E staining analysis of major organs and tumors revealed the high biocompatibility of NiOR (Figure S15).

\section{Discussion}

Arising from an imbalance between generation and elimination of ROS, oxidative stress has drawn increasing attention owing to its contribution to the development of various diseases. It is now recognized that redox regulation involving ROS is crucial to the modulation of critical cellular functions ${ }^{51}$. Underlying molecular response is complicated, but useful to understand critical pathology. "Off-line" methods such as western blotting and ELISA are available to analyze the changes under oxidative stress at the molecule level. However, such approaches cannot provide spatiotemporal information about the changes in living cells. Fluorescence imaging is an emerging "on-line" method to reflect the molecules in situ. Nanoparticle-based probes are desired to study the related targets in the fluorescence imaging of living because such probes are capable of carrying multiple reporters in a single assembly. Nevertheless, 
the unexpected interaction between reporters results in poor sensitivity and specificity. To data, there is no effective approach to administrate the molecular reporters.

Taking advantages of structural DNA nanotechnology, we integrated ROS reporter and APE1 into a single DNA scaffold (iOR). In iOR, the reporter distance was precisely controlled by MD simulation tool to enable minimum interaction. The relation between ROS and APE1 was disclosed in living cells and experimental mice by iOR and NiOR,. By analyzing the level of 8-oxo-dG, we found the molecular mechanism that elevated ROS level induces the overexpression of APE1 mainly because of the cumulative damaged bases in genomic DNA and mtDNA. Figure 5 shows the abundance of multiple targets under different oxidative stress implying APE1 and 8-oxo-dG are both proportional to ROS level, as well as nuclear and cytoplasmic APE1 are equally sensitive to ROS change. Moreover, we for the first time demonstrate that allicin a nature product from garlic exhibit great potential to mitigate oxidative stress, by spatiotemporal imaging of active small molecules and biological enzyme regulated oxidative stress.

In summary, a DNA scaffold-based molecular probe (iOR) was rationally designed by molecular dynamics simulation tool. It enables orthogonally reporting cellular ROS and its induced gene repair enzyme APE1. Owing to precisely controlled distance of the two reporters, cellular ROS and APE1 were analyzed with high specificity and sensitivity and spatiotemporal accuracy in living cells. Their cellular localization and abundance have been dissected, revealing that elevating oxidative stress can induce the expression level of APE1, the colocalization of ROS and APE1, and APE1's mitochondrial accumulation. This work demonstrates a generalizable strategy to construct orthogonal molecular reporters in an integrated nanostructure for dissecting related molecular targets in living cells and experimental animals.

\section{References}

1. Hultqvist, M., Olsson, L. M., Gelderman, K. A. \& Holmdahl, R. The protective role of ROS in autoimmune disease. Trends Immunol. 30, 201-208 (2009).

2. Reuter, S., Gupta, S. C., Chaturvedi, M. M. \& Aggarwal, B. B. Oxidative stress, inflammation, and cancer How are they linked? Free Radical Bio. Med. 49, 1603-1616 (2010).

3. Sosa, V. et al. Oxidative stress and cancer: An overview. Ageing Res. Rev. 12, 376-390 (2013).

4. Jenner, P. Oxidative stress in Parkinson's disease. Ann. Neurol. 533, S26-S36 (2003).

5. Wang, X. et al. Oxidative stress and mitochondrial dysfunction in Alzheimer's disease. Bba.-Mol. Basis Dis. 1842, 1240-1247 (2014).

6. Aimo, A. et al. Oxidative stress and inflammation in the evolution of heart failure: From pathophysiology to therapeutic strategies. Eur. J. Prev. Cardiol. 27, 494-510 (2020).

7. de Las Heras, N., Martin Gimenez, V. M., Ferder, L., Manucha, W. \& Lahera, V. Implications of Oxidative Stress and Potential Role of Mitochondrial Dysfunction in COVID-19: Therapeutic Effects of Vitamin D. 
ANTIOXIDANTS. 9, (2020).

8. Reuter, S., Gupta, S. C., Chaturvedi, M. M. \& Aggarwal, B. B. Oxidative stress, inflammation, and cancer How are they linked? Free Radical Bio. Med. 49, 1603-1616 (2010).

9. Nelson, B. C. \& Dizdaroglu, M. Implications of DNA damage and DNA repair on human diseases. Mutagenesis. 35, 1-3 (2020).

10. Yang, H. et al. A Photoresponsive Nanozyme for Synergistic Catalytic Therapy and Dual Phototherapy. Small. 17, (2021).

11. Wang, H. et al. Degradable Carbon-Silica Nanocomposite with Immunoadjuvant Property for DualModality Photothermal/Photodynamic Therapy. ACS Nano. 14, 2847-2859 (2020).

12. Borlinghaus, J., Albrecht, F., Gruhlke, M. C., Nwachukwu, I. D. \& Slusarenko, A. J. Allicin: chemistry and biological properties. Molecules. 19, 12591-12618 (2014).

13. Leung, K., Chakraborty, K., Saminathan, A. \& Krishnan, Y. A DNA nanomachine chemically resolves lysosomes in live cells. Nat. Nanotechnol. 14, 176 (2019).

14. Narayanaswamy, N. et al. A pH-correctable, DNA-based fluorescent reporter for organellar calcium. Nat. Methods. 16, 95-102 (2019).

15. Fu, S. et al. DNA nanotechnology enhanced single-molecule biosensing and imaging. TrAC-Trend. Anal. Chem. 140, (2021).

16. Ke, Y., Castro, C. \& Choi, J. H. Structural DNA Nanotechnology: Artificial Nanostructures for Biomedical Research. Annu. Rev. Biomed. Eng. 20, 375-401 (2018).

17. Fu, S. et al. Engineering high-robustness DNA molecular circuits by utilizing nucleases. Nanoscale. $12,6964-6970$ (2020).

18. Wang, C. et al. Rational Design of DNA Framework-Based Hybrid Nanomaterials for Anticancer Drug Delivery. Small. 16, e2002578 (2020).

19. Zhang, Y. et al. Probing and regulating the activity of cellular enzymes by using DNA tetrahedron nanostructures. Chem. Sci. 10, 5959-5966 (2019).

20. Li, F. et al. DNA nanotechnology-empowered nanoscopic imaging of biomolecules. Chem. Soc. Rev. 50, 5650-5667 (2021).

21. Han, Z. et al. Ultrasensitive detection of mRNA in extracellular vesicles using DNA tetrahedron-based thermophoretic assay. Nano Today. 38, (2021). 
22. Peshavariya, H. M., Dusting, G. J. \& Selemidis, S. Analysis of dihydroethidium fluorescence for the detection of intracellular and extracellular superoxide produced by NADPH oxidase. Free Radical Res. 41, 699-712 (2007).

23. Li, M. \& Wilson, D. M. I. Human Apurinic/Apyrimidinic Endonuclease 1. Antioxid. Redox Sign. 20,678707 (2014).

24. Antoniali, G. et al. Mammalian APE1 controls miRNA processing and its interactome is linked to cancer RNA metabolism. Nat. Commun. 8, 797 (2017).

25. Zhang, H. et al. Graphene Quantum Dot-Based Nanocomposites for Diagnosing Cancer Biomarker APE1 in Living Cells. ACS Appl Mater Interfaces. 12, 13634-13643 (2020).

26. Zhang, Y. et al. Probing and regulating the activity of cellular enzymes by using DNA tetrahedron nanostructures. Chem. Sci. 10, 5959-5966 (2019).

27. Li, L. et al. Base excision repair-inspired DNA motor powered by intracellular apurinic/apyrimidinic endonuclease. Nanoscale. 11, 1343-1350 (2019).

28. Liu, N. DNA Nanotechnology Meets Nanophotonics. Nano Lett. 20, 8430-8431 (2020).

29. Doye, J. P. K. et al. Coarse-graining DNA for simulations of DNA nanotechnology. Phys. Chem. Chem. Phys. 15, 20395-20414 (2013).

30. Ouldridge, T. E. et al. Optimizing DNA nanotechnology through coarse-grained modeling: a two-footed DNA walker. ACS Nano. 7, 2479-2490 (2013).

31. Snodin, B. E. et al. Direct Simulation of the Self-Assembly of a Small DNA Origami. ACS Nano. 10, 1724-1737 (2016).

32. Shi, Z., Castro, C. E. \& Arya, G. Conformational Dynamics of Mechanically Compliant DNA Nanostructures from Coarse-Grained Molecular Dynamics Simulations. ACS Nano. 11, 4617-4630 (2017).

33. Son, H., Mo, W., Park, J., Lee, J. \& Lee, S. Single-Molecule FRET Detection of Sub-Nanometer Distance Changes in the Range below a 3-Nanometer Scale. BIOSENSORS-BASEL. 10, (2020).

34. Wang, C. et al. Rational Design of DNA Framework-Based Hybrid Nanomaterials for Anticancer Drug Delivery. Small. 16, e2002578 (2020).

35. Su, X. et al. Simultaneous Fluorescence Imaging of the Activities of DNases and 3 ' Exonucleases in Living Cells with Chimeric Oligonucleotide Probes. Anal. Chem. 85, 9939-9946 (2013).

36. Ding, $\mathrm{H}$. et al. DNA Nanostructure-Programmed Like-Charge Attraction at the Cell-Membrane Interface. ACS CENTRAL SCIENCE. 4, 1344-1351 (2018). 
37. Yang, J. et al. Self-Assembled Double-Bundle DNA Tetrahedron for Efficient Antisense Delivery. ACS Appl Mater Interfaces. 10, 23693-23699 (2018).

38. Yang, C., Uertz, J., Yohan, D. \& Chithrani, B. D. Peptide modified gold nanoparticles for improved cellular uptake, nuclear transport, and intracellular retention. Nanoscale. 6, 12026-12033 (2014).

39. Reczek, C. R. \& Chandel, N. S. ROS Promotes Cancer Cell Survival through Calcium Signaling. Cancer Cell. 33, 949-951 (2018).

40. Angkeow, P. et al. Redox factor-1: an extra-nuclear role in the regulation of endothelial oxidative stress and apoptosis. Cell Death Differ. 9, 717-725 (2002).

41. Ozaki, M., Suzuki, S. \& Irani, K. Redox factor-1/APE suppresses oxidative stress by inhibiting the rac1 GTPase. Faseb J. 16, 889-890 (2002).

42. Li, M. et al. Human AP endonuclease/redox factor APE1/ref-1 modulates mitochondrial function after oxidative stress by regulating the transcriptional activity of NRF1. Free Radic Biol Med. 53, 237-248 (2012).

43. Barchiesi, A., Wasilewski, M., Chacinska, A., Tell, G. \& Vascotto, C. Mitochondrial translocation of APE1 relies on the MIA pathway. Nucleic Acids Res. 43, 5451-5464 (2015).

44. Li, N. et al. A DNA Tetrahedron Nanoprobe with Controlled Distance of Dyes for Multiple Detection in Living Cells and in Vivo. Anal. Chem. 89, 6670-6677 (2017).

45. Oushiki, D. et al. Development and application of a near-infrared fluorescence probe for oxidative stress based on differential reactivity of linked cyanine dyes. J. Am. Chem. Soc. 132, 2795-2801 (2010).

46. Si, F., Liu, Y., Yan, K. \& Zhong, W. A mitochondrion targeting fluorescent probe for imaging of intracellular superoxide radicals. Chem. Commun. 51, 7931-7934 (2015).

47. Dai, J., Chen, Y. \& Jiang, F. Allicin reduces inflammation by regulating ROS/NLRP3 and autophagy in the context of A. fumigatus infection in mice. Gene. 762, 145042 (2020).

48. Hong, Y., Nan, B., Wu, X., Yan, H. \& Yuan, Y. Allicin alleviates acrylamide-induced oxidative stress in BRL-3A cells. Life Sci. 231, 116550 (2019).

49. Xiang, Q. et al. Allicin Attenuated Advanced Oxidation Protein Product-Induced Oxidative Stress and Mitochondrial Apoptosis in Human Nucleus Pulposus Cells. Oxid. Med. Cell. Longev. 2020, 6685043 (2020).

50. Li, R. Q. et al. A two-photon fluorescent probe for exogenous and endogenous superoxide anion imaging in vitro and in vivo. Biosens. Bioelectron. 87, 73-80 (2017). 
51. Scandalios, J. G. Oxidative stress responses-what have genome-scale studies taught us? Genome Biol. 3, S1019 (2002).

\section{Methods}

\section{Reagents and materials.}

All the oligonucleotides used in this work were synthesized and purified by high-performance liquid chromatography (HPLC), (Sangon Co., Shanghai, China), their sequences are summarized in Table S1 and the use of these strands is explained in the denotation. Dihydroethidium (DHE) and 1-Ethyl-3-(3(dimethylaminopropyl carbodiimide hydrochloride (EDC) was purchased from Aladdin. (Shanghai, China). APE1, lambda exonuclease ( $\lambda$ exo), deoxyribonuclease I (DNase I), exonuclease I (Exo I), T7 exonuclease (T7 Exo), T5 exonuclease (T5 Exo), and their corresponding buffers were obtained from NEB (Ipswich, MA). 区-Tubulin (11H10) Rabbit mAb was purchased by Cell Signaling Technology Co. (USA), and APE1 mouse antibody was from Abcom (UK). DNase/RNase free deionized water from Tiangen Biotech Co. (Beijing, China) was used in all experiments. Xanthine (XA), xanthine oxidase (XO), phorbol 12-myristate 13-acetate (PMA), lipopolysaccharide (LPS) were obtained from Solarbio (Beijing, China). Hela, A549, HEK-293 cells were purchased from Procell Life Science \& Technology Co., Ltd. (Wuhan, China). All the chemicals were analytical grade, and ultrapure water with a resistivity of $18.2 \mathrm{M} \Omega \mathrm{cm}$ was used in all experiments.

Cell Counting Kit-8 (CCK-8) was from Dojindo Laboratories, Kumamoto (Japan). Reactive oxygen species (ROS) detection kit was purchased from Solarbio Co. (Beijing, China). 8-oxo-dG detection kit was purchased from Meimian Co. (Jiangsu, China). Anti-Rabbit Detection Module for Wes, Peggy Sue or Sally Sue and 12-230 kDa Wes Separation Module capillary cartridges were obtained from ProteinSimple Co. (USA).

\section{oxDNA simulation of iOR.}

The distance between fluorophores on DNA scaffold was analyzed by oxDNA, which is a coarse-grained MD simulation software program. In our cases, PolyT sequences of different lengths were used to construct different iOR candidates. The initial DNA structure was generated from oxView (https://sulcgroup.github.io/oxdna-viewer/), then two simulation files "example.top" and "example.dat" were used for oxDNA running program. The above-mentioned simulation files need to be "relax" in oxDNA to produce initial structure simulation files for Virtual Movement Monte Carlo (VMMC) model. After each initial structure was generated as expected, the molecular behavior of the DNA nanostructures was simulated by sequence-dependent Virtual move Monte Carlo conditions without mutual trapping. The specific parameters for VMMC simulation are shown in Table S2. During the simulation, DNA conformations over time were written down in "trajectory.dat". In these cases, the conformations were recorded every 10 steps and total simulation step was 100000 . Therefore, 10000 data points can be extracted from trajectory through a python script named "distance.py" in oxdna-analysis tool. The distance from the base modified Cy 5 to the base modified DHE was measured in each recorded 
conformation. Obtained data were used to chart and analyze, and the best structure of iOR can be obtained.

\section{Preparation of iOR and NiOR.}

To assemble iOR, $1 \mu \mathrm{M}$ of oligonucleotides (P1, P2, P3, P4, P5, P6, P7 Table S1) were mixed in 1×TM buffer ( $20 \mathrm{mM}$ Tris, $50 \mathrm{mM} \mathrm{MgCl}_{2}, \mathrm{pH}=8.0$ ), and denatured at $80{ }^{\circ} \mathrm{C}$ for $10 \mathrm{~min}$ followed by cooling to $4{ }^{\circ} \mathrm{C}$ in 30 min by using PCR thermocycler. DHE was conjugated to carboxyl-labeled P7 strand by EDC chemistry, and the conjugates were purified by centrifugation at $12000 \mathrm{rpm}$ for $5 \mathrm{~min}$ by ultrafiltration devices $(3 \mathrm{k}, 0.5 \mathrm{~mL})$ to remove unreacted DHE. To obtain NiOR, Alkyne-labeled NLS peptide (NDDEATADSQHSTPPKKKRKVEDPKDFPSEPra-C) was coupled to azide-labeled P8 strand using click chemistry. P7-HE and P8-NLS were characterized by $12.5 \%$ native PAGE which was operated at $4{ }^{\circ} \mathrm{C}$ for $45 \mathrm{~min}$ at a constant voltage of $120 \mathrm{~V}$, and the gel was subsequently stained with SYBR Gold. P8-NLS was also characterized by positive ion MALDI mass spectroscopy.

\section{Characterization of iOR and NiOR by gel electrophoresis, DLS and AFM.}

Native PAGE (6\%) was used to characterize the assembly process of DNA nanostructures. The gel was subsequently stained with SYBR Gold. To characterize the tetrahedron by AFM, a drop of $25 \mathrm{nM}$ nanostructures was spotted onto freshly cleaved mica surface and kept for $10 \mathrm{~s}$ to achieve strong adsorption. The sample drop was then washed off by $1 \times$ TM buffer and dried by compressed air. DNA samples were imaged by tapping-mode AFM (FastScan, Bruker) with OTESPA-R3 tips. The tip-surface interaction was minimized by optimizing the scan set-point. Particle size was also measured by DLS (NanoZS90, Malvern Instruments, U.K.) at $25^{\circ} \mathrm{C}$.

\section{Testing the probe reactivity by fluorescence.}

iOR was used for all in vitro fluorescence assays. In order to test the response of $\mathrm{O}_{2}{ }^{\cdot-}$, the XO/XA system was used to generate $\mathrm{O}_{2}{ }^{\cdot-}$ ( 1 unit of XO can catalyze $1 \mathrm{~mol}$ of $\mathrm{XA}$ to yield $330 \mathrm{nM} \mathrm{O}_{2}{ }^{-{ }^{-}}$) in PBS ${ }^{44} .100 \mathrm{nM}$ of iOR was mixed with various concentrations of XO/XA and were incubated at $37^{\circ} \mathrm{C}$ for $45 \mathrm{~min}$, then the fluorescence emission profile was recorded $(\lambda e x: 488 \mathrm{~nm}$ ). Fluorescence dequenching assay of iOR was used to detect the activity of APE1. $100 \mathrm{nM}$ of iOR was mixed with various concentrations of APE1 at 37 ${ }^{\circ} \mathrm{C}$, then the fluorescence emission profile of Cy 5 was recorded ( $\lambda$ ex: $625 \mathrm{~nm}$ ). Enzymatic kinetics of multiple enzymes $(5 \mathrm{U} / \mathrm{mL}$ APE1, $1 \mathrm{U} / \mathrm{mL}$ DNase I, $0.4 \mathrm{U} / \mathrm{mL}$ Exo I, $2 \mathrm{U} / \mathrm{mL} \lambda$ Exo, $2.5 \mathrm{U} / \mathrm{mLT} 5$ Exo, $2 \mathrm{U} / \mathrm{mLT7}$ Exo) was measured by fluorescence kinetics at $37^{\circ} \mathrm{C}$ on a real-time PCR thermocycler (RotorGene Q, QIAGEN, Germany). 10 mM EDTA (final concentration) was used to stop the reaction. The products were characterized by $6.0 \%$ native PAGE.

\section{Cell culture and fluorescence imaging.}

Hela, A549, HEK-293 were cultured in the complete medium at $37{ }^{\circ} \mathrm{C}$ in a humidified atmosphere of $5 \%$ $\mathrm{CO}_{2} / 95 \%$ air (for details see Table S3). Cells were transferred to a laser confocal culture dish with an 
appropriate density, incubated with mito-tracker $(200 \mathrm{nM})$ and nuclear staining dye Hoechst33324 $(10 \mu \mathrm{g} / \mathrm{mL})$. After removing the above dyes, the cells were incubated with $100 \mathrm{nM}$ of $\mathrm{NiOR}$. Highly inclined and laminated optical sheet (HILO) fluorescence microscopy was assembled by a Nikon inverted microscopy (ECLIPSE, Ti-U) carried with 100xmagnification, 1.49 numerical aperture (NA) TIRFM objective (Nikon), an EMCCD cameras (Ixon 897, Andor). For HILO illumination, the lasers of 488 and $647 \mathrm{~nm}$ were coupled into a homemade illuminator to excite DHE and Cy5, respectively. Optical filters (Semrock) of $590 \mathrm{~nm}$ and $670 \mathrm{~nm}$ were used to detect the corresponding emission. The fluorescence images were given to $0.15 \mu \mathrm{m}$ per pixel, and the images were acquired with an exposure time of $50 \mathrm{~ms}$ (gain 3). Mito-tracker and nuclear staining dye were excited by mercury light with appropriate filters. External stimuli were used to regulate ROS and APE1 (see the legend in Figure 3).

\section{Evaluation of cellular APE1 by western blotting.}

Protein quantitative analyses were performed according to supplier's protocol (ProteinSimple, USA). Cells were lysed on ice and then centrifuged. BCA Protein Assay Kit was used to determined protein concentrations. Western blotting was performed using a Wes Simple Western system, an automated capillary-based size sorting system (ProteinSimple, USA). Five micrograms of total protein from each

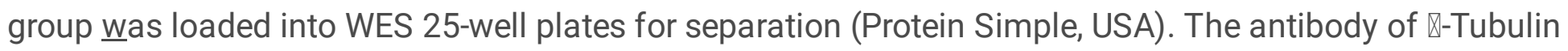
(dilution ratio was 1/100) and APE1 (dilution ratio was 1/100) were used as primary antibody. The electrophoretic separation and immunological detection parts were taken place in the capillary system automatically.

\section{Detection of human 8-oxo-dG in cell extracts using an enzyme-linked immunosorbent assay kit (ELISA).}

All of the cells were collected and broken by high-throughput ultrasonic disruptor in $1 \times$ PBS. The supernatant was collected, and centrifugation with $12000 \mathrm{rpm}$ was performed at $4{ }^{\circ} \mathrm{C}$ for $20 \mathrm{~min}$ to remove bio-macromolecules. The supernatants were detected by 8-oxo-dG ELISA kit (MMBIO, China). The absorbance of $450 \mathrm{~nm}$ was recorded to reflect the 8-oxo-dG abundance.

\section{Animals experiments and in vivo fluorescence imaging.}

BALB/c nude mice were from Servicebio Co., Ltd. (Wuhan, China). They were raised in a specific pathogen-free animal facility and had access to water and food. The animal experiment protocols were approved by the Animal Ethics Committee of the Beijing University of Chemical Technology. HeLa tumor cells were inoculated under the armpits of mice until the tumor volume reached $100 \mathrm{~mm}^{3}$. Table S4 shows the feed administration of each group in Figure 4. NiOR $(10 \mu \mathrm{M}, 25 \mu \mathrm{L})$ was injected into the tumor site of mice by using a micro syringe. Mice were imaged at predetermined time intervals by in vivo fluorescence imaging system (IVIS). After injection of the probe for $24 \mathrm{~h}$, The tumors and major organs of mice were isolated and fixed in $4 \%$ paraformaldehyde solution. They were paraffin-embedded, deparaffinized, rehydrated, and then Hematoxylin and Eosin (H\&E) staining and TdT-mediated dUTP NickEnd Labeling (TUNEL) was performed. Immunofluorescence was performed for APE1 with a dilution ratio of antibody of $1 / 100$. 


\section{Data Processing and statistical Analysis}

The cell fluorescence images were analyzed by ImageJ 8.0. The colocation of fluorescence is analyzed by 'Colocalization' and 'plot profile' module, while 'Measure' module is used to analyze mean fluorescence intensity of cells. Compass software (ProteinSimple, USA) was used to analyze the relative amount of each protein in western blotting, through the areas under peaks from the chemiluminescence chromatograms. Living Image Software 4.4 for IVIS Lumina XR Series $₫$ was used to analyze the fluorescence imaging results of mice. Bar charts, line plots and heat map were made by GraphPad 8.0.6. Radar Diagrams (Figure 5) were made by Excel 2016. One-way ANOVA was conducted using IBM SPSS Statistics 23 according to Duncan's test $(p<0.05)$.

\section{Declarations}

\section{Acknowledgement}

This work was supported by Modern Agricultural Industry Technology System, National Project for Risk Assessment of Agro-product Quality and Safety, Innovation project of Chinese Academy of Agricultural Sciences, State Key Research Development Program of China (2019YFC1200500, 2019YFC1200502), National Natural Science Foundation of China (31971361), and the Natural Science Foundation of Beijing Municipality (5212013).

\section{Author Contribution}

X.S., R.W., and Y.Q. conceived the ideas and designed the study. Y.P., R.W., X.W., G.L., J.Q., and Z.Q. designed, performed and analysed the experiments. L.Z. completed the molecular simulation. X.S., R.W., Y.P., R.L., and X.D. wrote the manuscript, with critical revision by J.Q. and Y.Q. Y.Q. provided technical support and advice. All the authors read and approved the final version of the manuscript.

\section{Competing interests}

There is no conflict to declare.

\section{Data availability}

The data that supports the plots within this paper and other finding of this study are available from the corresponding author upon reasonable request.

\section{Supplementary information}

Supplementary Figure S1-15, Supplementary Table 1-4.

\section{Scheme}


Scheme 1 is available in the Supplementary Files section.

\section{Figures}

(A)

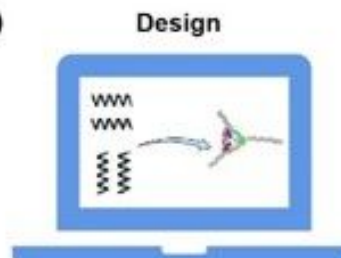

(B) $\quad \begin{array}{ccc}\text { Distance }(\mathrm{nm}) \\ 0 & 10 \quad 20 & 300\end{array}$

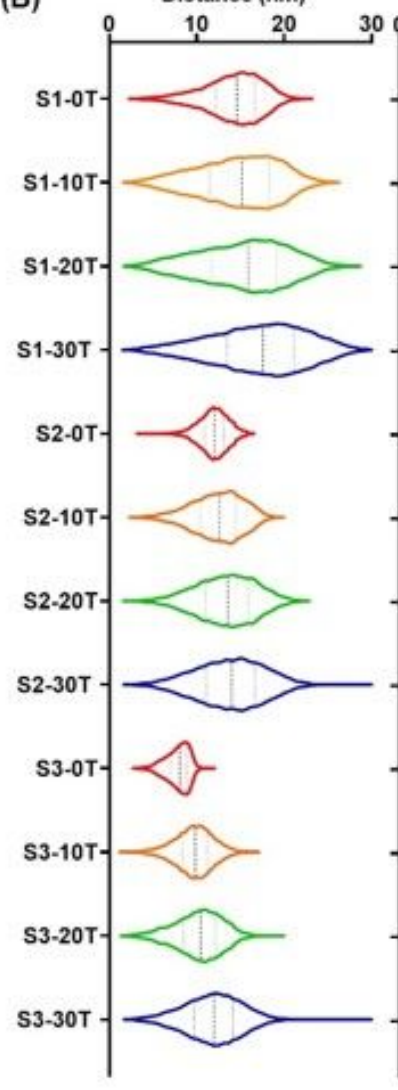

(F)

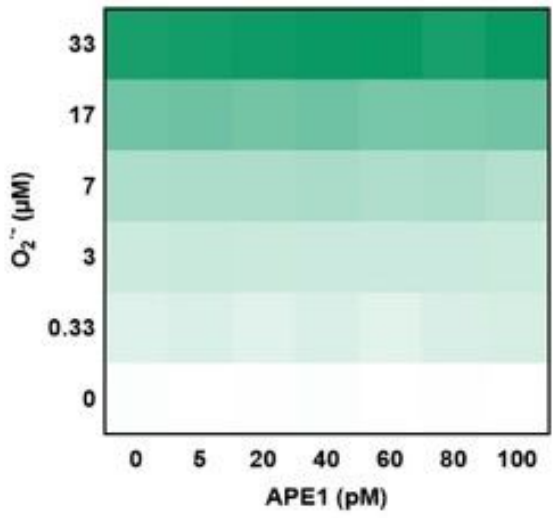

Simulation

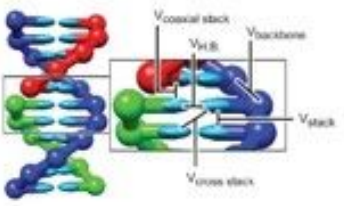

FRET Probability (\%)

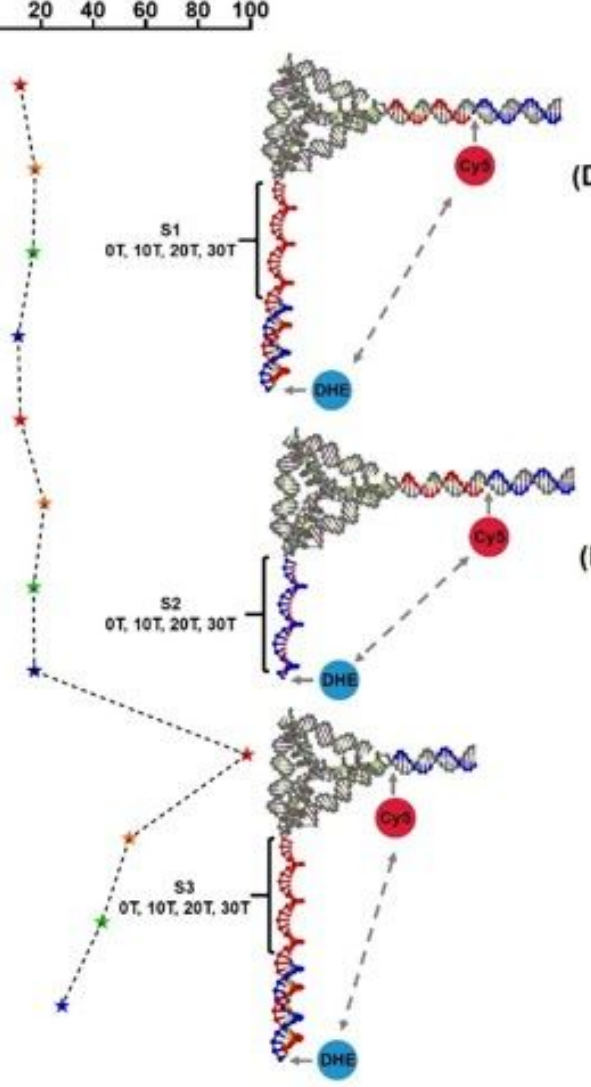

(E)

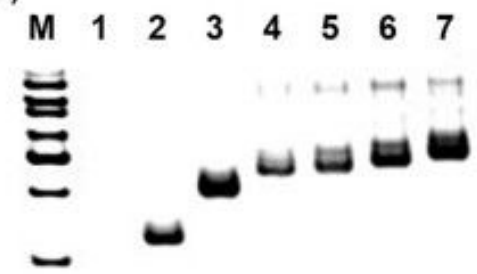

D)
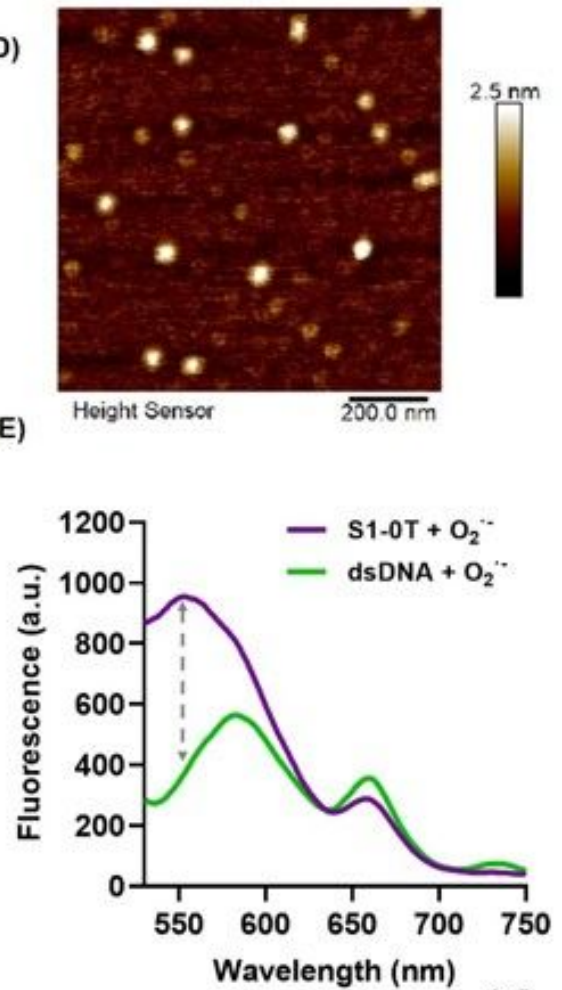

DHE
(a.u.)
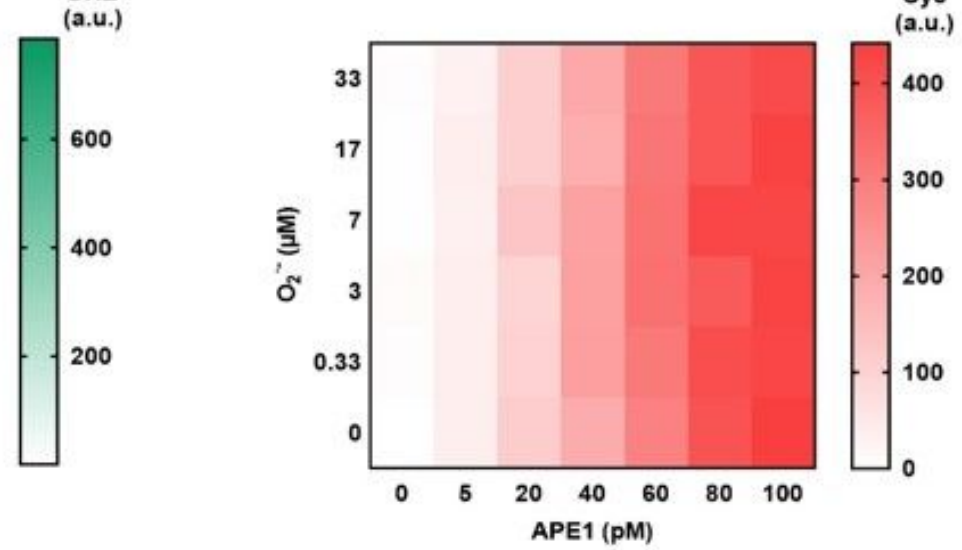

Figure 1 
Simulation and characterization of iOR. (A) The pipeline of iOR design and simulation. (B) MD simulation results of IOR. The positions of DHE and Cy 5 dyes were marked in iOR candidates. Distance distribution of the two dyes in different structures, and the probability of FRET (>10\%) was shown. (C) The characterization of the stepwise assembly of iOR by $6.0 \%$ PAGE. lane 1: P1, lane 2: P1+P2, lane 3 : $\mathrm{P} 1+\mathrm{P} 2+\mathrm{P} 3$, lane 4: $\mathrm{P} 1+\mathrm{P} 2+\mathrm{P} 3+\mathrm{P} 4$, lane 5: $\mathrm{P} 1+\mathrm{P} 2+\mathrm{P} 3+\mathrm{P} 4+\mathrm{P} 5$, lane 6: $\mathrm{P} 1+\mathrm{P} 2+\mathrm{P} 3+\mathrm{P} 4+\mathrm{P} 5+\mathrm{P} 6$, lane 7 : $\mathrm{P} 1+\mathrm{P} 2+\mathrm{P} 3+\mathrm{P} 4+\mathrm{P} 5+\mathrm{P} 6+\mathrm{P} 7-\mathrm{DHE}$. (D) AFM characterization of iOR (scale bar $100 \mathrm{~nm}$ ). (E) Fluorescence emission profiles of the optimized iOR (S1-0T) and the undesired iOR (S3-0T) in the presence of ROS. (F) One-pot dual-iOR detection of ROS and APE1 with various concentration combinations. All tests were performed with three biological replicates. 
(A)

(B)

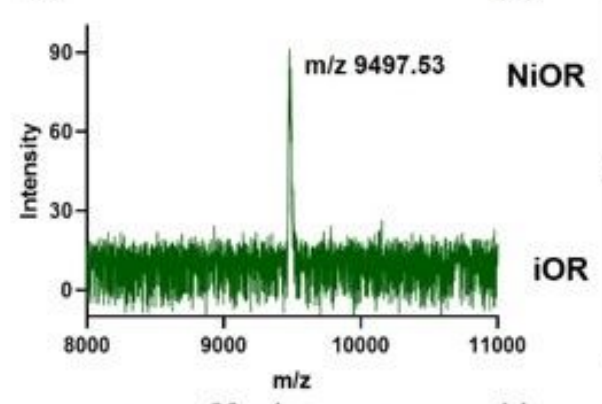

(D)
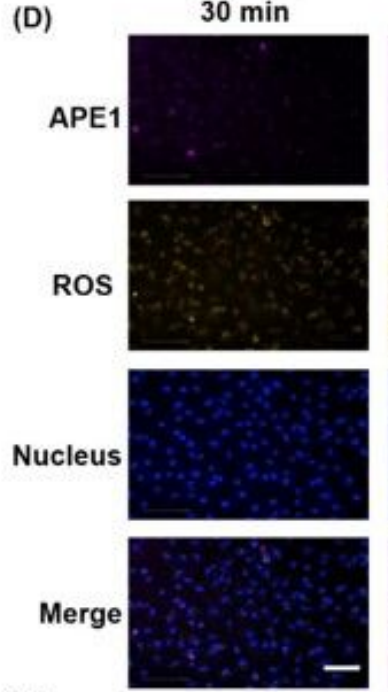

(E)

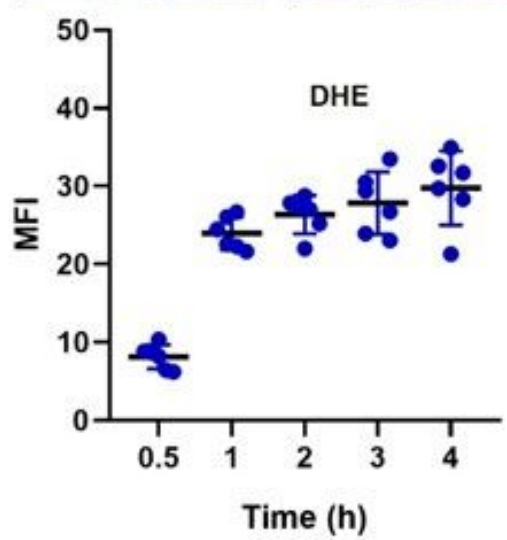

(C)
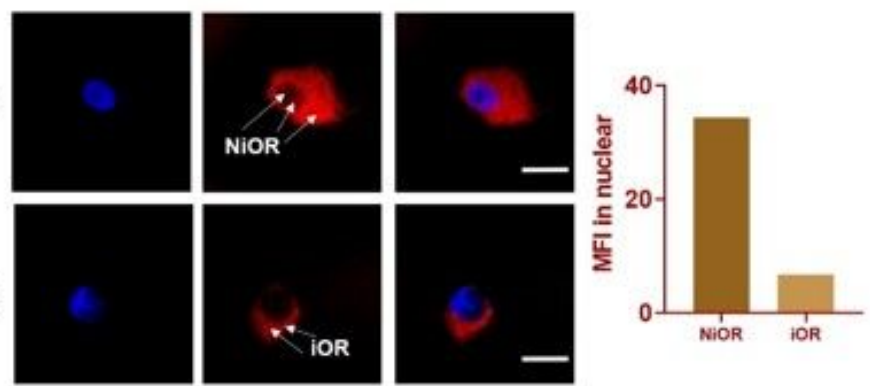

$3 \mathrm{~h}$
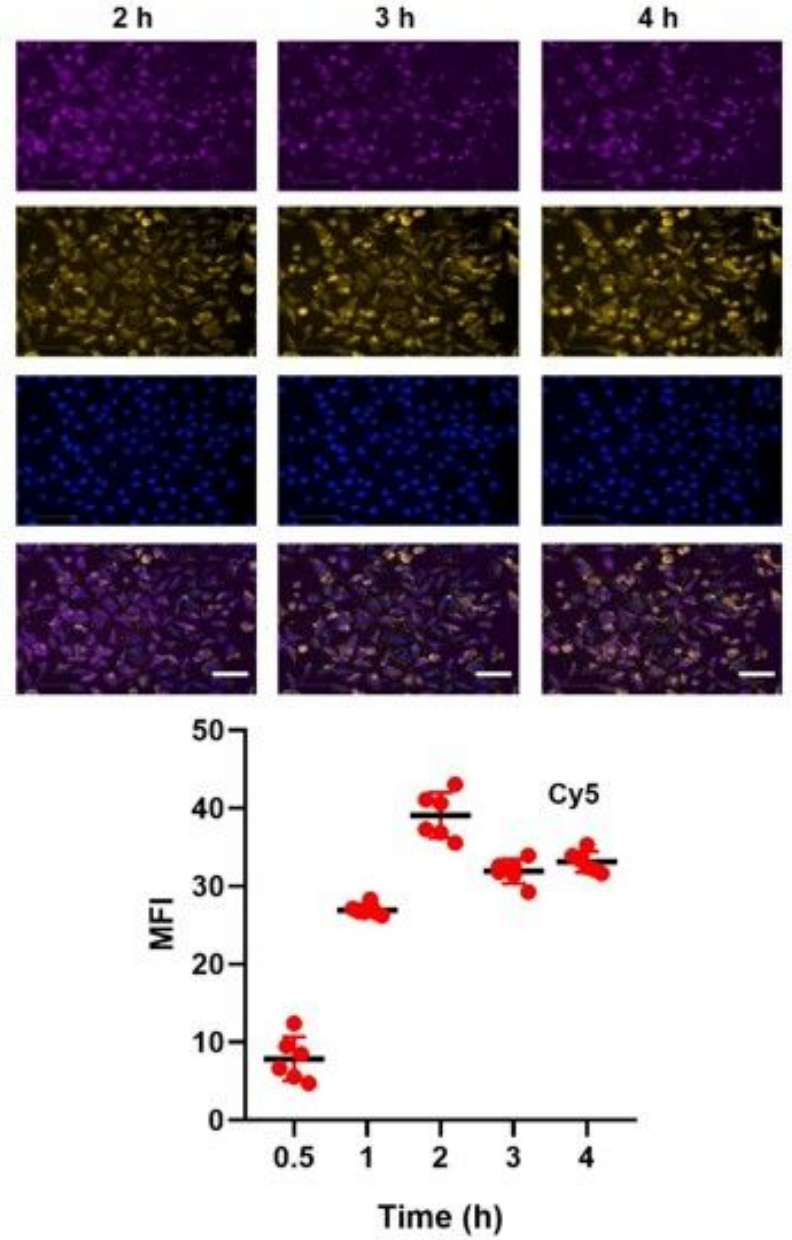

Figure 2

NLS functionalized iOR (NiOR) preparation and its internalization. (A) NLS functionalization of iOR preparation by click chemistry. (B) Positive ion MALDI mass spectrum of oligonucleotide-peptide conjugates. The signals corresponding to singly protonated forms of the and the conjugate product $(\mathrm{m} / \mathrm{z}$ 9497.53) are labeled in the spectrum. (C) Cellular distribution of NiOR and iOR which is singly labeled by Cy5. Both probes distributed in cytoplasm thoroughly, and only NiOR exhibits nuclear distribution. The 
ratio of nuclear and cytoplasmic fluorescence was shown. Scale bar: $20 \mu \mathrm{m}$. (D) Internalization process and reaction with cellular ROS and APE1 of NiOR in HeLa cells. The nucleus was stained by Hoechst33324 (scale bar=20 $\mu \mathrm{m}$ ). (E) Mean fluorescence intensity of DHE and Cy 5 in cells as function of time. Each data set represents a single cell.

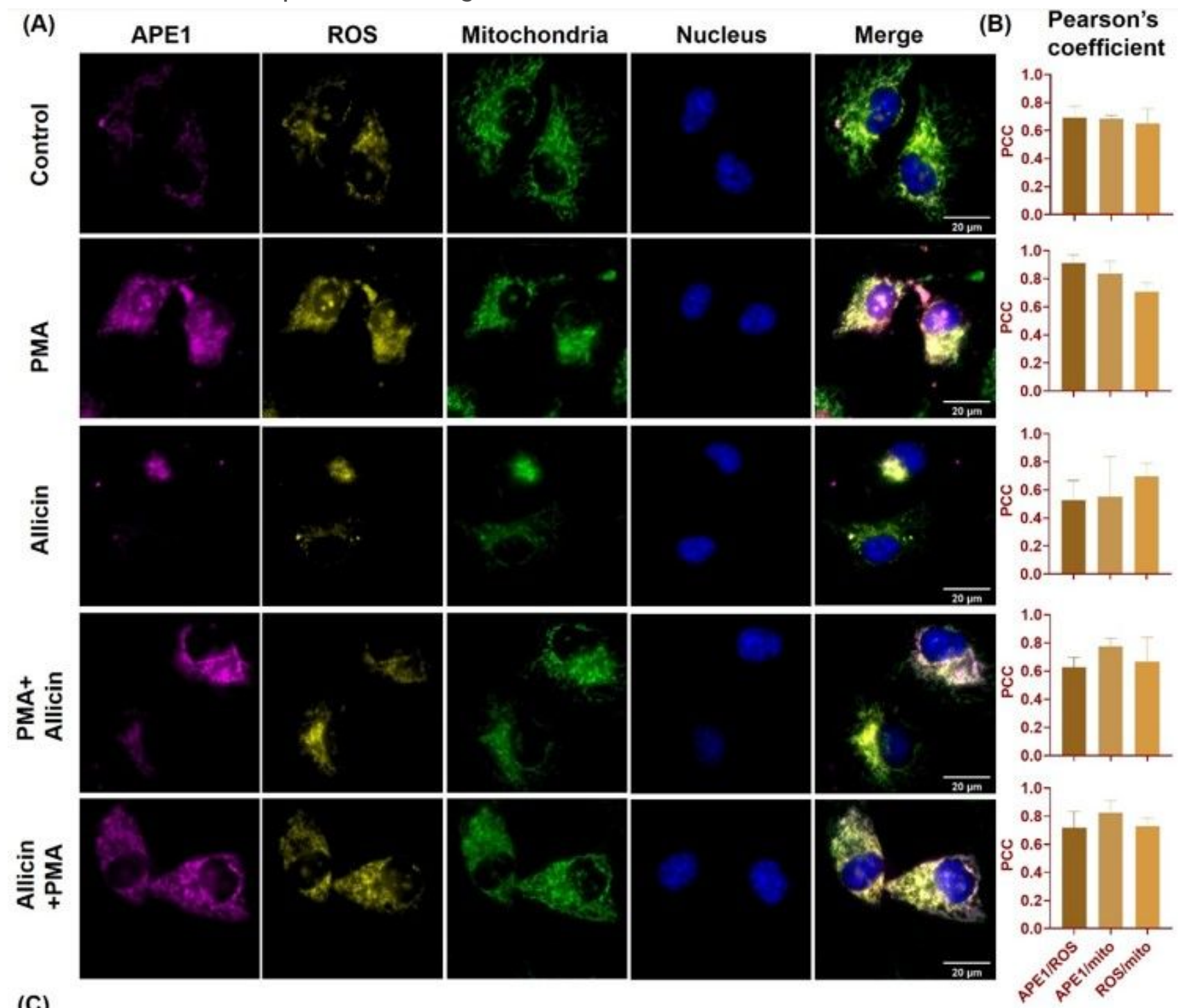

(C)

Nuclear ROS

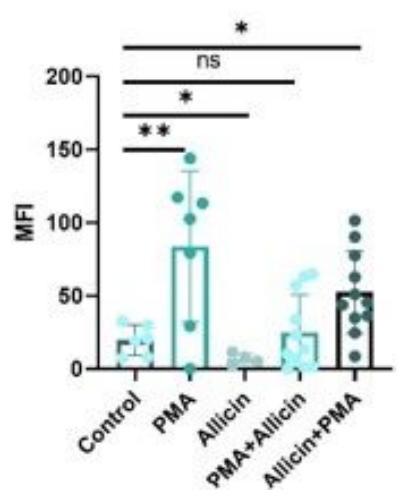

Figure 3
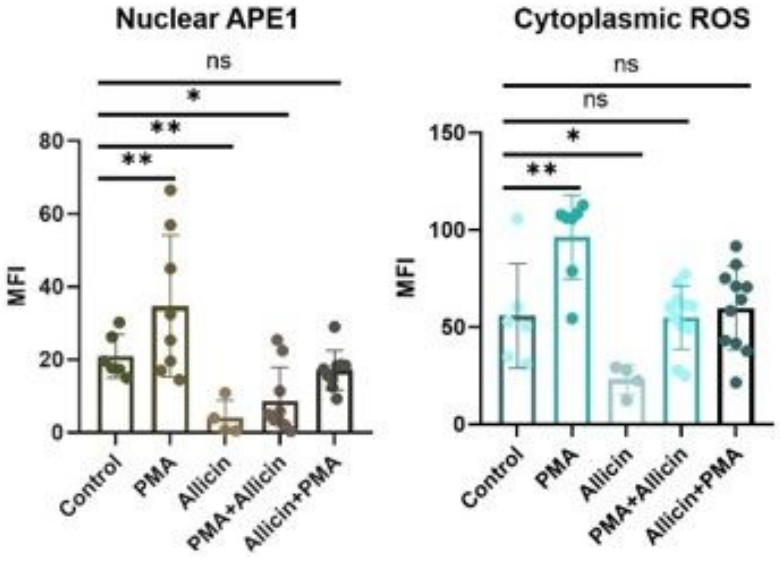

\section{Cytoplasmic APE1}

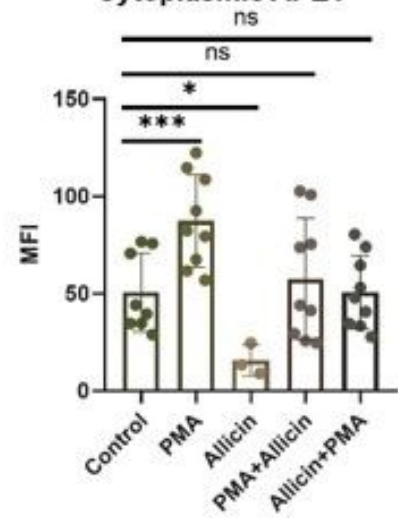


Analysis of the abundance and subcellular distribution of ROS and APE1 in HeLa cell line under different oxidative stress. (A) Fluorescence images of ROS and APE1 by using NiOR, mitochondria and nucleus were stained by mito-tracker and Hoechst33324. Control, cells had no oxidative stimulation; Allicin, cells were treated with allicin (10 mg/L) for $24 \mathrm{~h}$; PMA, cells were stimulated with PMA (500 mg/L) for $30 \mathrm{~min}$; PMA + Allicin: cells were treated with PMA $(500 \mathrm{mg} / \mathrm{L})$ for $30 \mathrm{~min}$ followed by Allicin $(10 \mathrm{mg} / \mathrm{L})$ for $24 \mathrm{~h}$; Allicin + PMA: cells were treated with allicin $(10 \mathrm{mg} / \mathrm{L})$ for $24 \mathrm{~h}$ followed by PMA $(500 \mathrm{mg} / \mathrm{L})$ for 30 min. Scale bar: $20 \mu \mathrm{m}$. (B) Pearson's correlation coefficient (PCC) is used to indicate the co-localization of dual-channel fluorescence. (C) Mean fluorescence intensity (MFI) from single cells. ${ }^{\star \star \star} p<0.001, \star \star p<0.01$, * $p<0.05$, ns $p>0.05$. 
(A)

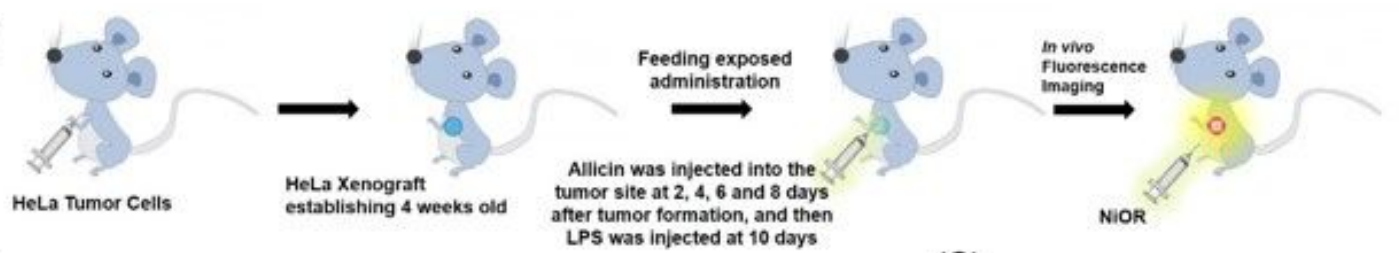

(B)
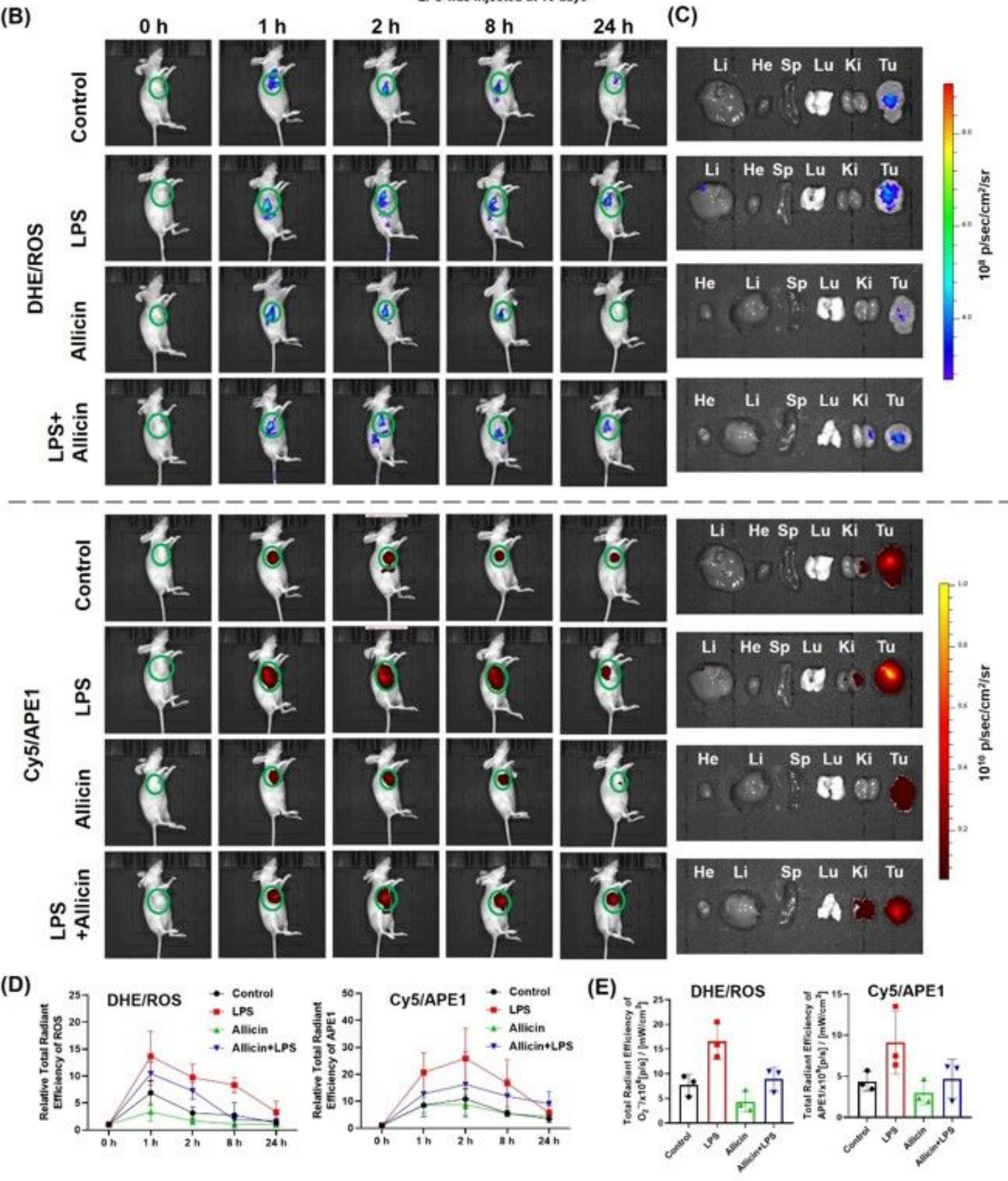

Figure 4

In vivo fluorescence imaging of ROS and APE1 by NiOR. (A) Schematic illustration of HeLa tumor xenograft establishment, NiOR and external stimuli administration modalities. (B) In vivo fluorescence imaging at different times after the injection of NiOR. Circles indicate the tumor sites. (C) Ex vivo fluorescence imaging of the major organs (He: heart, Li: liver, Sp: spleen, Lu: lung, and Ki: kidney) and 
tumors (Tu) that harvested from the euthanized mice at $24 \mathrm{~h}$ post-injection. (D) Average signal intensity of tumor site. (E) Average signal intensity of harvested tumor, for major organs see Figure S12.

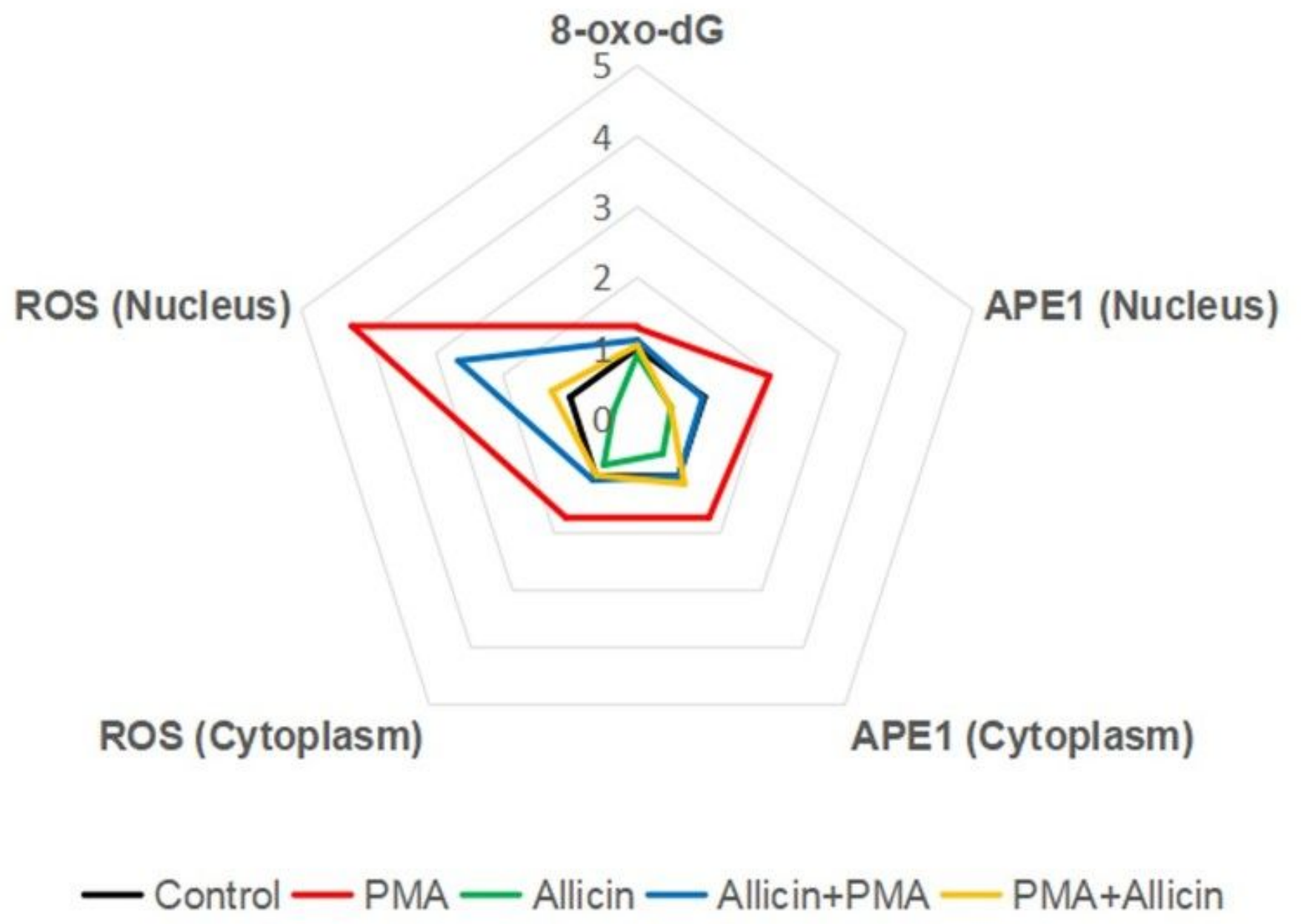

Figure 5

Radar diagram of relative ROS, APE1, and 8-oxo-dG levels under different oxidative stress. ROS and APE1 were measured by NiOR fluorescence imaging (Figure $2 \mathrm{~A}$ and $2 \mathrm{C}$ ), and 8-oxo-dG was measured by ELISA (Figure S9(B)). Each species in the control group was normalized as 1 . The pentagonal area can represent the variation of multispecies among groups.

\section{Supplementary Files}

This is a list of supplementary files associated with this preprint. Click to download.

- Scheme1.jpg

- PanetalIntramolecularOrthogonalReportersforDissectingOxidativeStressandResponseSI.pdf 